\title{
Dose Dependent Antimicrobial Cellular Cytotoxicity - Implications for ex vivo Diagnostics
}

\author{
Ana Copaescu ${ }^{1 * t}$, Phuti Choshi ${ }^{2 \dagger}$, Sarah Pedretti ${ }^{2}$, Effie Mouhtouris ${ }^{1}$, Jonathan Peter ${ }^{2,3 \neq}$ and \\ Jason A. Trubiano ${ }^{1,4,5,6 \neq}$ \\ ${ }^{1}$ Centre for Antibiotic Allergy and Research, Department of Infectious Diseases, Austin Health, Heidelberg, VIC, Australia, ${ }^{2}$ Allergy \\ and Immunology Unit, University of Cape Town Lung Institute, Cape Town, South Africa, ${ }^{3}$ Division of Allergy and Clinical \\ Immunology, Department of Medicine, University of Cape Town, Cape Town, South Africa, ${ }^{4}$ Department of Oncology, Sir Peter \\ MacCallum Cancer Centre, The University of Melbourne, Parkville, VIC, Australia, ${ }^{5}$ Department of Medicine (Austin Health), The \\ University of Melbourne, Heidelberg, VIC, Australia, ${ }^{6}$ The National Centre for Infections in Cancer, Peter MacCallum Cancer \\ Centre, Melbourne, VIC, Australia
}

\section{OPEN ACCESS}

Edited by: Roberto Paganelli, University of Studies G. d'Annunzio Chieti and Pescara, Italy

Reviewed by:

Sudheer Kumar Ravuri, Steadman Philippon Research

Institute, United States Khalid Muhammad, United Arab Emirates University,

United Arab Emirates

*Correspondence: Ana Copaescu ana.copaescu@gmail.com

${ }^{\dagger}$ These authors share first authorship

${ }^{\ddagger}$ These authors share senior authorship

Specialty section:

This article was submitted to Translational Pharmacology, a section of the journal Frontiers in Pharmacology

Received: 01 March 2021

Accepted: 30 July 2021

Published: 10 August 2021

Citation:

Copaescu A, Choshi P, Pedretti S, Mouhtouris E, Peter $J$ and Trubiano JA (2021) Dose Dependent Antimicrobial Cellular Cytotoxicity -Implications for ex vivo Diagnostics.

Front. Pharmacol. 12:640012. doi: 10.3389/fphar.2021.640012
Introduction: Ex vivo and in vitro diagnostics, such as interferon- $\gamma$ (IFN- $\gamma$ ) release enzyme linked ImmunoSpot (ELISpot) and flow cytometry, are increasingly employed in the research and diagnostic setting for severe T-cell mediated hypersensitivity. Despite an increasing use of IFN- $\gamma$ release ELISpot for drug causality assessment and utilization of a range of antimicrobial concentrations ex vivo, data regarding antimicrobial-associated cellular cytotoxicity and implications for assay performance remain scarcely described in the literature. Using the measurement of lactate dehydrogenase $(\mathrm{LDH})$ and the 7-AAD cell viability staining, we aimed via an exploratory study, to determine the maximal antimicrobial concentrations required to preserve cell viability for commonly implicated antimicrobials in severe T-cell mediated hypersensitivity.

Method: After an 18-h incubation of patient peripheral blood monocytes (PBMCs) and antimicrobials at varying drug concentrations, the cell cytotoxicity was measured in two ways. A colorimetric based assay that detects LDH activity and by flow cytometry using the 7-AAD cell viability staining. We used the PBMCs collected from three healthy control participants with no known history of adverse drug reaction and two patients with a rifampicin-associated drug reaction with eosinophilia and systemic symptoms (DRESS), confirmed on IFN- $\gamma$ ELISpot assay. The PBMCs were stimulated for the investigated drugs at the previously published drug maximum concentration (Cmax), and concentrations 10and 100-fold above.

Results: In a human immunodeficiency virus (HIV) negative and a positive rifampicinassociated DRESS with positive ex vivo IFN- $\gamma$ ELISpot assay, use of 10- and 100-fold Cmax drug concentrations decreased spot forming units/million cells by $32-100 \%$, and this corresponded to cell cytotoxicity of more than 40 and $20 \%$ using an LDH assay and

\footnotetext{
Abbreviations: Cmax, maximum concentration recorded; CTL, cytotoxic T-cells; DRESS, drug reaction with eosinophilia and systemic symptoms; EDTA, ethylenediaminetetraacetic acid; ELISpot, enzyme linked immunospot; FBS, fetal bovine serum; IFN- $\gamma$, interferon- $\gamma$; LDH, lactate dehydrogenase; PBMCs, peripheral blood mononuclear cells; SCARs, severe cutaneous
} adverse reactions. 
7-AAD cell viability staining, respectively. The other antimicrobials (ceftriaxone, flucloxacillin, piperacillin/tazobactam, and isoniazid) tested in healthy controls showed similar dose-dependent increased cytotoxicity using the LDH assay, but cytotoxicity remained lower than $40 \%$ for all Cmax and 10-fold Cmax drug concentrations except flucloxacillin. All 100-fold Cmax concentrations resulted in cell death $>40 \%$ (median 57\%), except for isoniazid. 7-AAD cell viability staining also confirmed an increase in lymphocyte death in PBMCs incubated with 10-fold and 100-fold above Cmax for ceftriaxone, and flucloxacillin; however, piperacillin/tazobactam and isoniazid indicated no differences in percentages of viable lymphocytes across concentrations tested.

Conclusion: The LDH cytotoxicity and 7-AAD cell viability staining techniques both demonstrate increased cell death corresponding to a loss in ELISpot sensitivity, with use of higher antimicrobial drug concentrations for ex vivo diagnostic IFN- $\gamma$ ELISpot assays. For all the antimicrobials evaluated, the use of Cmax and 10-fold Cmax concentrations impacts cell viability and potentially affects ELISpot performance. These findings inform future approaches for ex vivo diagnostics such as IFN- $\gamma$ release ELISpot.

Keywords: delayed hypersensitivity reaction, drug allergy, severe cutaneous adverse reaction, T-cell, enzyme linked ImmunoSpot, cytotoxicity, flow cytometry, lactate dehydrogenase

\section{INTRODUCTION}

T-cell mediated adverse drug reactions can be lifethreatening, with mortality rates up to $50 \%$ in certain severe cutaneous adverse drug reactions (SCAR) (Peter et al., 2017). Antimicrobial drugs are responsible for a significant burden of these reactions; more so in high burden tuberculosis (TB) and human immunodeficiency virus (HIV) settings (Blumenthal et al., 2019). Delayed hypersensitivity reactions to antimicrobials frequently occur in the setting of multidrug regimens e.g., TB infection; and rapid, accurate, and safe diagnostic tools to aid the identification and exclusion of the offending drug are critical (Lehloenya et al., 2020). Drug provocation testing is often considered contraindicated in most settings of severe, life-threatening adverse drug reactions; while systemic reactions to in vivo diagnostics such as patch testing has been reported, especially in the context of HIV infection (Lehloenya et al., 2020). Thus, in vitro and ex vivo diagnostics are appealing and have been increasingly employed in the evaluation of presumed T-cell mediated hypersensitivity reactions (Lehloenya et al., 2020).

The Enzyme-Linked ImmunoSpot (ELISpot) assay, an ex vivo diagnostic, was initially introduced in 1983 (Sedgwick and Holt, 1983) and detects locally secreted cytokine molecules, such as IFN- $\gamma$, using antibody-coated plates offering both a qualitative but also a quantitative specific protein measurement, measuring cytokine production on a per-cell basis (Cox et al., 2006). Successful IFN- $\gamma$ production requires adequate, live $\mathrm{T}$ lymphocytes. Despite an increasing use of IFN- $\gamma$ release ELISpot for drug causality assessment and utilization of a range of antimicrobial concentrations ex vivo, data regarding antimicrobial-associated cellular cytotoxicity and implications for assay performance remain scarcely described in the literature.

\section{MATERIALS AND METHODS}

We used the peripheral blood monocytes (PBMCs) from three control participants and two patients with IFN- $\gamma$ ELISpot confirmed rifampicin-associated drug reaction with eosinophilia and systemic symptoms (DRESS). To understand the required blood quantity for each assay, one needs to keep in mind that, for a healthy control, $9 \mathrm{ml}$ of blood gives us approximately $1 \times 10^{7}$ PBMC. About $3 \times 10^{7}$ (or $27 \mathrm{ml}$ of blood) is required for each executed assay (e.g., ELISpot, LDH, or flow cytometry).

For these patients, the blood was collected less than 2 weeks after their acute DRESS reaction and they were not known to have any other drug hypersensitivities. PBMCs were isolated from heparinized peripheral blood [ethylenediaminetetraacetic acid (EDTA) tubes] by density gradient centrifugation (using polysaccharide Ficoll Paque $^{\circledR}$ ) as described previously (Trubiano et al., 2018) and preserved in liquid nitrogen or a $-80^{\circ}$ freezer until assessment. After thawing, prior to sample use, cell viability was measured using $0.4 \%$ trypan blue staining solution (Thermo Fisher Scientific ${ }^{\circledR}$ ) and the Countess ${ }^{\circledR}$ automated cell counter (Fisher Scientific, 2010).

\section{IFN- $\gamma$ ELISpot Assay}

The INF- $\gamma$ ELISpot assay was performed using a negative (unstimulated, media alone) and a positive control (antihuman CD3 antibody, 1-D1K, Mabtech $^{\circledR}$ ). The drug concentrations used for ELISpot and corresponding to the Cmax and the 10-fold value are illustrated in Supplementary Table S1. The average number of spots for the test and unstimulated wells were calculated. A positive response was defined as equal or greater than 50 spot forming units (SFU)/ million cells after background (unstimulated control) removal as per previously published definitions (Keane et al., 2012; 
TABLE 1 | Drug concentrations used for LDH assay and flow cytometry using the 7-AAD viability stain on lymphocytes.

\begin{tabular}{lccc} 
Drugs & \multicolumn{3}{c}{ Concentrations $(\boldsymbol{\mu g} \mathbf{g} \mathbf{m l})$} \\
\cline { 2 - 4 } & $\boldsymbol{\# 1}$ & $\mathbf{\# 2}$ & $\mathbf{\# 3}$ \\
\hline Ceftriaxone & $\mathbf{2 0 0}$ & $\mathbf{2 , 0 0 0}$ & 20,000 \\
Flucloxacillin & $\mathbf{2 0 0}$ & $\mathbf{2 , 0 0 0}$ & 20,000 \\
Piperacillin-tazobactam & $\mathbf{1 5 0 / 1 8 . 7 5}$ & $\mathbf{1 , 5 0 0 / 1 8 7 . 5}$ & $15,000 / 1,875$ \\
Isoniazid & $\mathbf{5 0}$ & $\mathbf{5 0 0}$ & 5,000 \\
Rifampicin & $\mathbf{2 5}$ & $\mathbf{2 5 0}$ & 2,500
\end{tabular}

Note: The concentrations in bold characters are the ones currently used for the ELISpot assay.

Keane et al., 2014). The method is illustrated in Supplementary Figure S1. Spots were analyzed automatically with an AID ELISpot Reader (software version 7.0) (Mabtech $^{\circledR}$ ).

\section{Drug Concentrations for Cytotoxicity Assays}

The penicillin derivatives were provided by the local hospital pharmacies (Austin Health, Australia, and University of Cape Town Private Hospital, South Africa): ceftriaxone $(50 \mathrm{mg} / \mathrm{ml})$, flucloxacillin $(50 \mathrm{mg} / \mathrm{ml})$, and piperacillin-tazobactam $(200 \mathrm{mg} /$ $\mathrm{ml})$. The remaining drugs were commercially bought products: isoniazid (10 mg/ml; Sigma ${ }^{\circledR}$, I3377-50G) and rifampicin $(60 \mathrm{mg} /$ ml; Sigma ${ }^{\circledR}$, R3501-250 MG). The cells $\left(2 \times 10^{5} /\right.$ well $)$ were added in triplicates for the control patients and duplicates for the two DRESS patients to three pre-determined drug concentrations: including Cmax, 10-fold above and 100-fold above (Table 1) and then allowed to incubate for $18 \mathrm{~h}$ at $37^{\circ} \mathrm{C}$ with $5 \% \mathrm{CO}_{2}$. The initial concentration tested for flucloxacillin and ceftriaxone was $200 \mu \mathrm{g} /$ $\mathrm{ml}, 150 \mu \mathrm{g} / \mathrm{ml}$ for piperacillin-tazobactam and $25 \mu \mathrm{g} / \mathrm{ml}$ for isoniazid and rifampicin (Table $\mathbf{1}$ ).

\section{Cytotoxicity Based Lactate Dehydrogenase Assay}

Cytotoxicity or cell death is measured with assays using markers of apoptosis and/or necrosis of target cells (Saade et al., 2012; Specian et al., 2016). The colorimetric measurement of lactate dehydrogenase $(\mathrm{LDH})$, is a reliable and commonly used technique (Specian et al., 2016). LDH, the cytosolic enzyme present in nucleated cells, is released into the extracellular media once the cell membrane is disrupted. The LDH activity is measured indirectly by the transformation of lactate in pyruvate with the reduction of nicotinamide adenine dinucleotide $\left(\mathrm{NAD}^{+}\right)$to $\mathrm{NAD}^{+} \mathrm{H}^{+}$lactate (Specian et al., 2016; Abcam, 2019). The oxidation of the $\mathrm{NADH}^{+}$that causes an increase in absorbance will be proportional to the $\mathrm{LDH}$ activity and therefore proportional to the number of lysed cells (Saade et al., 2012).

The LDH assay was performed according to the manufacturer's suggested protocol (Abcam, 2019). H-Cytotox, 2019 (LDH Assay, Abcam ${ }^{\circledR}$ kit: ab65393). After addition of LDH reaction mix, an optimal $30 \mathrm{~min}$ incubation was used. The optical density of the plate was read with a FLUOstar Optima plate reader set at $450 \mathrm{~nm}$ with a $620 \mathrm{~nm}$ reference wavelength using the Optima version 2.1 software (BMG Labtech ${ }^{\circledR}$ ). The percentage of cytotoxicity was calculated as per equation in Supplementary Figure S2. GraphPad Prism version 8.0 software was used for data analysis (GraphPad Software, La Jolla California United States, www.graphpad.com).

\section{Flow Cytometry Using the 7-AAD Cell Viability Staining}

Lymphocyte viability (Saade et al., 2012) was assessed with the use of flow cytometry using the fluorescent DNA intercalator 7-AAD (7-aminoactinomycin). 7-AAD is a fluorescent derivative of actinomycin D that selectively binds to GC regions of the DN, exposed during cell death; and is frequently used to stain and exclude dead cells in flow cytometry (Zembruski et al., 2012).

Cryopreserved PBMCs were thawed and rested in R10 media at $37^{\circ} \mathrm{C}$ for $3 \mathrm{~h}$. Cells $\left(2-3 \times 10^{5}\right)$ were incubated for $18 \mathrm{~h}$ with antimicrobials tested. After incubation, cells were stained with CD3 Alexa Fluor 700 and CD4 PerCp Cy5.5 (BD Pharmingen) and CD8-APC AF750 (Invitrogen) for $30 \mathrm{~min}$ at $4^{\circ} \mathrm{C}$, then washed with FACS buffer (PBS containing 1\% bovine serum albumin and $0.05 \%$ sodium azide). After washing, PBMCs were stained with 7-AAD (BD Pharmingen) for $15 \mathrm{~min}$. Cell events were acquired on a LSRII flow cytometer and FlowJo software was used to analyse FCS files. The gating strategy is detailed in Supplementary Figure S3.

This study was approved by both the Austin Health ethics committee and the Human Research Ethics committee of the University of Cape Town. The investigators obtained written informed consent from the participants.

We sought to determine the maximal antimicrobial concentrations for cell viability using the cytotoxicity based $\mathrm{LDH}$ assay and the 7-AAD cell viability staining for commonly implicated antimicrobials in severe T-cell mediated hypersensitivity-ceftriaxone, flucloxacillin, piperacillintazobactam, isoniazid, and rifampicin.

\section{RESULTS}

\section{Effects of Increasing Cytotoxicity on ELISpot Performance in Rifampicin DRESS Patient}

Figures 1A,B show the SFU/million cells for the two DRESS patients with rifampicin as the offending drug (one HIV negative Figure 1A and one HIV co-infected Figure 1B). The SFU/million cells are highest at the lowest rifampicin concentrations ( $\mathrm{Cmax}=$ $25 \mathrm{mg} / \mathrm{ml}$, SFU/million cells of 673 and 187) and decrease in both patients at the 10 -fold drug concentration to 268 (60\% reduction) and 128 (32\% reduction) respectively. At drug concentrations of 100 -fold Cmax there are no T cell making IFN- $\gamma$ and thus no SFU/million (100\% reduction). Figures 1C,D show how cytotoxicity, measured by either the $\mathrm{LDH}$ assay or 7-AAD staining, increases with the 10- and 100-fold Cmax drug concentrations. In both the HIV negative and positive 
A

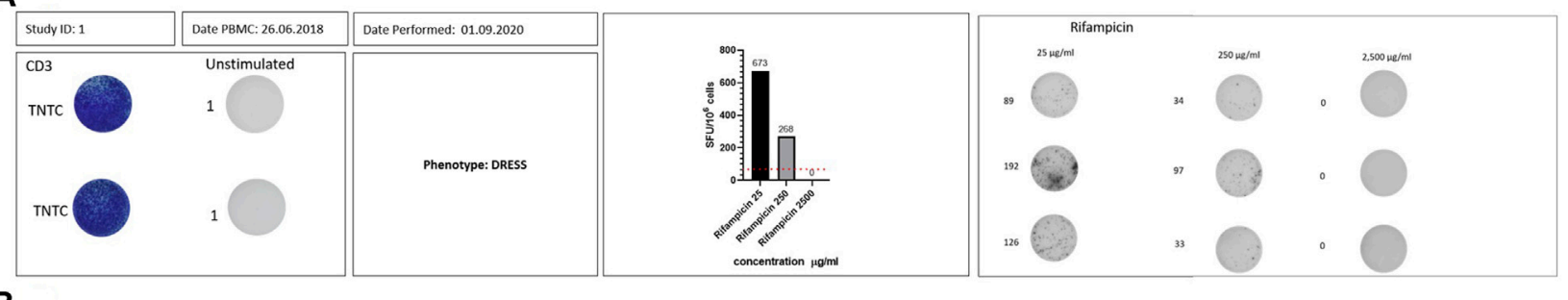

B
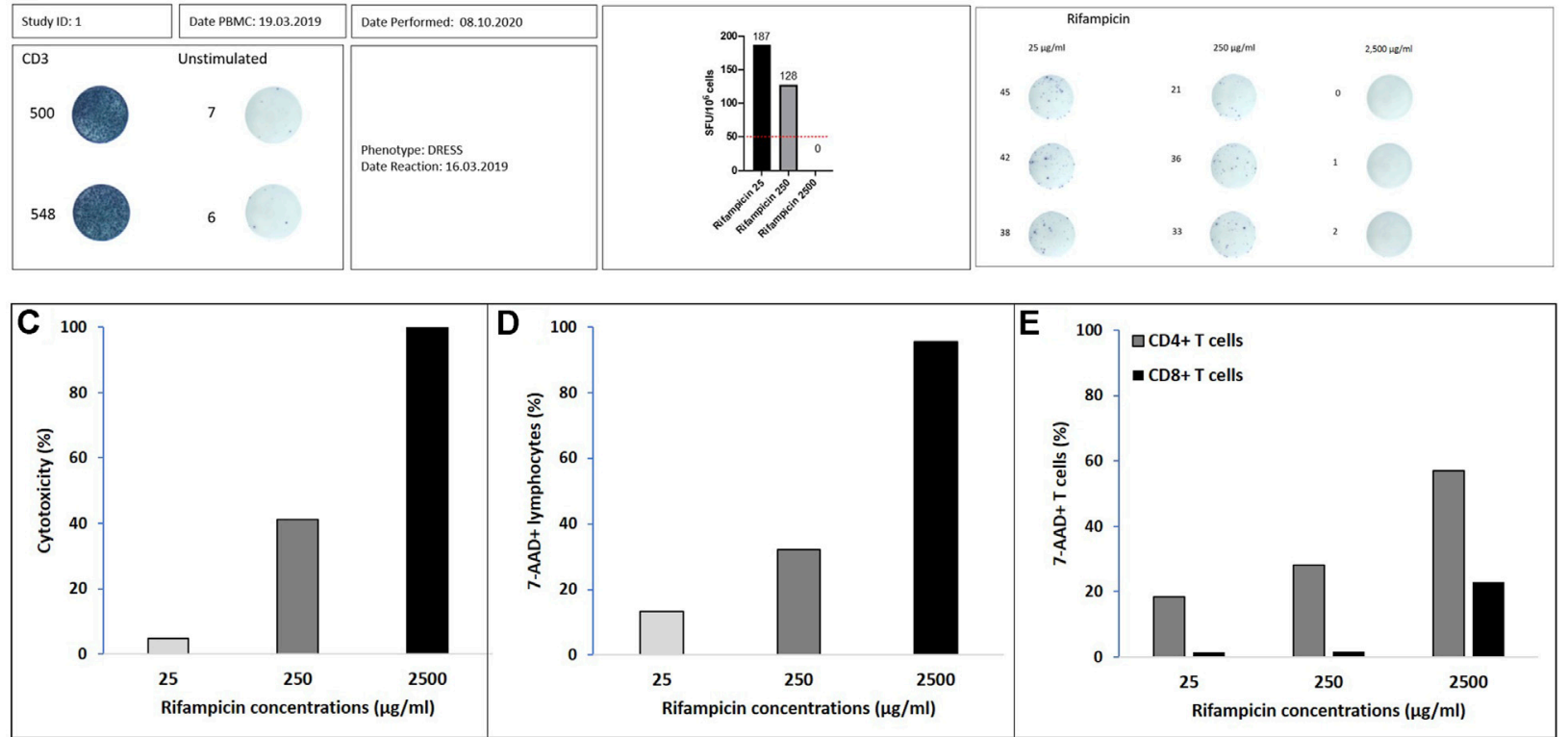

FIGURE 1 | Rifampicin DRESS patients. IFN- $\gamma$ ELISpot graphical illustration of (A) Australian patient and (B) south African patient. Positivity is defined by $\geq 50$ spot forming unit (SFU)/million cells after background (unstimulated control) removal (dotted line on the corner right figures). Positive controls were done using CD3 and the unstimulated wells represent media alone. The stimulations with the different drug concentrations $(\mu \mathrm{g} / \mathrm{ml})$ were done in triplicates. The numbers adjacent to the wells represent the number of spots measured with the ELISpot reader. ELISpot graphical illustration for Rifampicin 25, 250, and $2500 \mu \mathrm{g} / \mathrm{ml}$. Percentage of cytotoxicity assessed by (C) LDH assay, (D) flow cytometry with 7-AAD viability staining in lymphocytes and (E) flow cytometry with 7-AAD viability staining in CD4 ${ }^{+}$and $C D 8^{+} T$ cells.

rifampicin-associated DRESS patients there was dose-dependent cytotoxicity with increasing concentrations of rifampicin. CD4 positive as well as CD8 positive lymphocytes showed the same pattern as illustrated in Figure 1E.

\section{Cytotoxicity Based LDH Assay}

The number of viable cells initially added to the assay was $2 \times 10^{5}$ per well, with cell recovery post thawing measured for all controls as follows: $\mathrm{C} 1,91 \%$; 2 , 76\%, and C3, 80 and 75\% for the patients that had a DRESS reaction to rifampin. The $\mathrm{T}$ cell activation in response to the offending drug for the patients was confirmed by a positive ELISpot assay as per previous definition (Figures 1A,B).

The percentage of cytotoxicity seen in the LDH assay for all antimicrobials tested increased in a dose-dependent manner with increasing concentrations, but the extent of cytotoxicity varying by drug. The average cytotoxicity results for the three normal controls are illustrated in Figure 2A. The lowest concentrations, corresponding to previously published Cmax values and also used in ex vivo methods for ELISpots, showed a mean value of $8.4 \%$
(SD 8.4) cellular death. The mean percentage of cell death at 10fold above Cmax was $23.1 \%$ (SD 20.8), but only rifampicin and flucloxacillin showed cytotoxicity more than $40 \%$. In contrast, at the 100-fold Cmax drug concentrations the mean cell death was $97.7 \%$ (SD 113.1), and only isoniazid had a cytotoxicity less than $40 \%$ at this supra-physiological concentration.

\section{Flow Cytometry Using the 7-AAD Cell Viability Staining}

The number of cells added to the assay was $3 \times 10^{5}$ per well. The immunological intolerance for the patient with rifampicin DRESS was confirmed by a positive ELISpot assay (Figure 1B).

In control participants, lymphocyte cytotoxicity assessed by flow cytometry using 7-AAD viability staining showed variations with increasing concentrations, but results were drug-dependent in this assay. The average cytotoxicity results for the three normal controls are illustrated in Figure 2B. The lowest concentrations, corresponding to previously published Cmax values and also used in ex vivo methods for ELISpots, showed a mean 7-AAD staining 

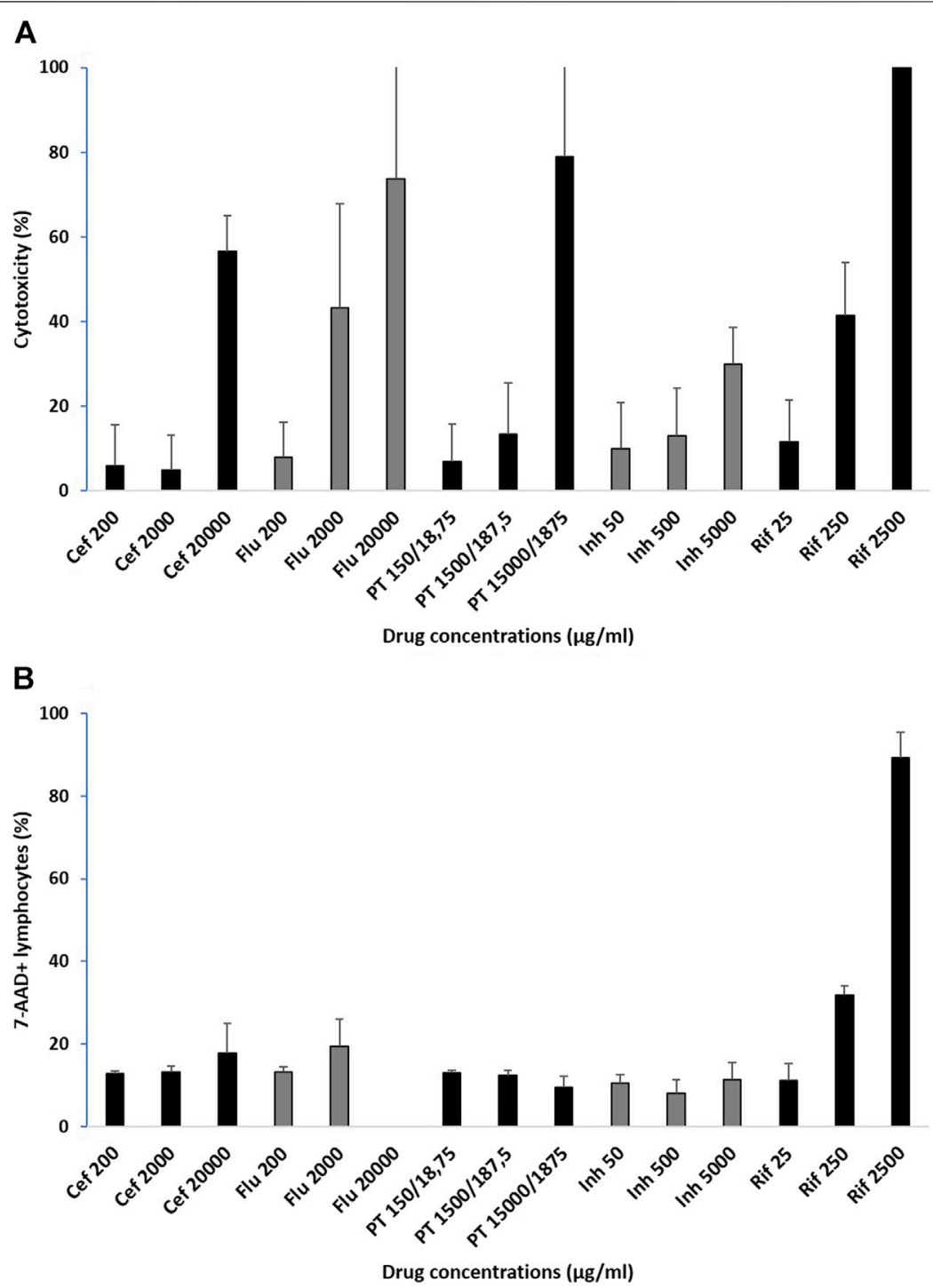

FIGURE 2 | Percentage of cytotoxicity in healthy controls assessed by (A) LDH assay and (B) flow cytometry with 7-AAD viability staining in lymphocytes. Cytotoxicity average for 3 normal controls for the drugs included in the study at 3 pre-determined drug concentrations: Cmax, concentrations 10-fold and 100-fold, mean \pm sd, Cef: ceftriaxone; Flu: flucloxacillin; PT: piperacillin-tazobactam; Inh: isoniazid; Rif: rifampicin.

of $11.9 \%$ (SD 2.4). For the 10 -fold above Cmax, the mean was $17.3 \%$ (SD 8.7), but only rifampicin had staining above $20 \%$ at this drug concentration. For the 100 -fold Cmax the mean 7-AAD staining was $31.9 \%$ (SD 35.1), with rifampicin very high. There was an increase in 7-AAD staining for PBMCs incubated with increasing concentrations of ceftriaxone, flucloxacillin (except the highest concentration which was not run due to technical difficulties) and rifampicin, no change with isoniazid and a slight decrease with piperacillin-tazobactam.

\section{DISCUSSION}

As IFN- $\gamma$ release ELISpot is increasingly used for drug causality assessment in patients with life-threatening, severe immune- mediated adverse drug reactions (IM-ADRs), ELISpot has been shown to be an efficient ex vivo tool in the diagnosis of antibiotic-associated SCAR patients (Trubiano et al., 2018) by offering the benefit of observing the patient's reaction to a drug without re-exposing the patient. To maximise assay sensitivity, it is advantageous to use the highest possible drug concentrations, especially in situations where the mechanism of drug-immune interaction is poorly defined. In this project, we examine antimicrobial-associated cellular cytotoxicity for common offending antimicrobials and its impact on assay performance. We describe two patients with rifampicin-associated DRESS (one HIV negative and one positive); as well as three control participants where the cytotoxicity of different antimicrobials used in clinical diagnostic was evaluated. Our main findings were: i) that in both the HIV negative and positive 
rifampicin-associated DRESS patients, there was a dosedependent reduction in SFU/million cells in the ELISpots corresponding to increasing rifampicin-induced drug cytotoxicity; ii) for all the antimicrobials evaluated, the use of Cmax and 10-fold Cmax concentrations did not reach cytotoxicity seen with rifampicin and are therefore unlikely to affect ELISpot performance; and iii) for drugs such as piperacillin/tazobactam and isoniazid even concentrations as high as 100 -fold Cmax did not result in cytotoxicity likely to reduce ELISpot performance.

ELISpot is an assay that requires optimization according to the population and drug of interest as well as the specific cytokine measured (Ji and Forsthuber, 2016). At high concentrations, drugs become toxic for cells via different mechanisms like overproduction of nitric oxide; generation of reactive oxygen species, and subsequent oxidative stress; mitochondrial dysfunction; and DNA damage. Not all drugs have equivalent cytotoxicity at the same concentrations and thus these experiments inform the optimization of diagnostic ELISpot for delayed IM-ADRs. Indeed, rifampicin appeared the most toxic of all tested drugs as illustrated with over $90 \%$ cytotoxicity at 100 fold Cmax concentrations and complete abrogation of SFUs in the ELISpot assay. Mechanisms of increased cytotoxicity with rifampicin are uncertain but the formation of drug-antibody complexes, which binds to platelet membrane activating complement and increasing cell death has been proposed (Combrink et al., 2020).

Various drug concentrations are described in the literature for ex vivo assays with varying units of measure including, millimoles (isoniazid, 1-4 mM; rifampicin, $0.1 \mathrm{mM}$ ) for tuberculosis related drugs (Usui et al., 2017) or micromoles (El-Ghaiesh et al., 2012) and $\mathrm{mg} / \mathrm{ml}$ (Rozieres et al., 2009; Tanvarasethee et al., 2013; Sedlackova et al., 2018) for beta-lactam drugs. Thus, a strength of this project is the use of the measured Cmax as a reference to ensure that these units are comparable across drugs and concentrations are optimised as being the point of maximal bacterial cytotoxicity with minimum tolerable cellular injury (Supplementary Table S1).

We noted higher percentage of cytotoxicity with the $\mathrm{LDH}$ assay compared with the 7-AAD staining method. The 7-AAD staining involves a nuclear dye, while the $\mathrm{LDH}$ assay measures the oxidation of the $\mathrm{NADH}^{+}$after cell membrane damage and $\mathrm{LDH}$ release into the extracellular medium. This distinction in assays could explain the difference observed in Figure 1. For the 7-AAD staining, all the drugs tested at the three concentrations, except rifampicin, showed relatively low cytotoxicity $(<30 \%)$ while for the LDH assay, most drug concentrations 10-fold higher than Cmax resulted notable increases in cytotoxicity (>40\%). The localisation of the damage-cytosolic ( $\mathrm{LDH}$ assay) versus nuclear (7-AAD) may explain these differences, but the correlation of both assay with decreased SFUs in the rifampicin ELISpot provides clear data linking the differing degrees of cytotoxicity with decreasing assay performance.

Using flow cytometry for evaluating cytotoxicity can be performed with the uptake of labeled annexin $\mathrm{V}$, propidium iodide or the uptake of fluorescent DNA probes 7-aminoactinomycin (7-AAD), as in our study (Saade et al., 2012). In apoptotic cells, membrane phosphatidylserine, which is located in the inner part of the membrane in healthy cells, translocates to the outer leaflet and is exposed to the external environment (Saade et al., 2012), where it binds with annexin V staining providing evidence of early cell death. Further, using 7-AAD dye gives information on loss of membrane integrity and later stages of cell death.

The study has limitations that include the inability to evaluate all the different drug concentrations for all antimicrobials in patients where the drug is known to be offending, and to directly match cytotoxicity data with ELISpot performance. However, the rifampicin data shows proof of concept and provides guidelines on when cytotoxicity levels are likely to effect assay performance. In addition, as with any laboratory assay, technical limits particularly for long-lasting cell cultures and complex manipulations as well as operator-dependent variability could influence results. Other potential considerations are sample irregularities in terms of time and condition of sample storage as well as drug formulation and preparation.

\section{CONCLUSION}

Our results show that the known Cmax for each antimicrobial and the 10-fold increased drug concentrations showed a mean percentage of cytotoxicity below $30 \%$, allowing a significant live population to evaluate in ex vivo experiments when these concentrations are employed. An increase in cytotoxicity was translated by fewer SFU in the ELISpot illustrating the impact of cell death on reduced ELISpot performance. These exploratory findings will inform approaches for ex vivo diagnostics such as IFN- $\gamma$ release ELISpot increasingly utilized in drug causality assessments of rare severe T-cell mediated hypersensitivity.

\section{DATA AVAILABILITY STATEMENT}

The original contributions presented in the study are included in the article/Supplementary Material, further inquiries can be directed to the corresponding author.

\section{ETHICS STATEMENT}

The studies involving human participants were reviewed and approved by both the Austin Health ethics committee and the Human Research Ethics committee of the University of Cape Town. The patients/participants provided their written informed consent to participate in this study.

\section{AUTHOR CONTRIBUTIONS}

PC and SP realized the laboratory research and employed the 7$\mathrm{AAD}$ assay as well as contributed to the manuscript text. EM and $A C$ realized the laboratory research and employed the $\mathrm{LDH}$ assay as well as contributed to the manuscript text. PC, SP, JP, JT, and 
AC analyzed the laboratory data and wrote the manuscript text. All authors reviewed the final manuscript text.

\section{FUNDING}

JP receives financial support from National Institutes of Health, award K43TW011178-02; the EDCTP2 programme supported by the European Union, grant number TMA2017SF-1981 and the South African National Research Foundation (NRF). PC receives a $\mathrm{PhD}$ fellowship from the Fogarty HATTP program, funded by the National Institutes of Health, award D43

\section{REFERENCES}

Abcam (2019). LDH-cytotoxicity Assay Kit II. Available from: https://www.abcam. com/ps/products/65/ab65393/documents/ab65393\%20LDH\%20Cytotoxicity\% 20Assay\%20Kit\%20II\%20Protocol\%20v4\%20(website).pdf (cited 07 28, 2020).

Blumenthal, K. G., Peter, J. G., Trubiano, J. A., and Phillips, E. J. (2019). Antibiotic Allergy. The Lancet 393 (10167), 183-198. doi:10.1016/s0140-6736(18)32218-9

Combrink, M., Loots, D. T., and du Preez, I. (2020). Metabolomics Describes Previously Unknown Toxicity Mechanisms of Isoniazid and Rifampicin. Toxicol. Lett. 322, 104-110. doi:10.1016/j.toxlet.2020.01.018

Cox, J., Ferrari, G., and Janetzki, S. (2006). Measurement of Cytokine Release at the Single Cell Level Using the ELISPOT Assay. Methods 38 (4), 274-282. doi:10.1016/j.ymeth.2005.11.006

El-Ghaiesh, S., Monshi, M. M., Whitaker, P., Jenkins, R., Meng, X., Farrell, J., et al. (2012). Characterization of the Antigen Specificity of T-Cell Clones from Piperacillin-Hypersensitive Patients with Cystic Fibrosis. J. Pharmacol. Exp. Ther. 341 (3), 597-610. doi:10.1124/jpet.111.190900

Fisher Scientific, T. (2010). "Assays for Cell Viability, Proliferation and Function," in Molecular Probes ${ }^{\mathrm{TM}}$ Handbook.

Ji, N., and Forsthuber, T. G. (2016). ELISPOT Techniques. Methods Mol. Biol. 1304, 63-71. doi:10.1007/7651_2014_111

Keane, N. M., Pavlos, R. K., McKinnon, E., Lucas, A., Rive, C., Blyth, C. C., et al. (2014). HLA Class I Restricted CD8+ and Class II Restricted CD4+ T Cells Are Implicated in the Pathogenesis of Nevirapine Hypersensitivity. AIDS 28 (13), 1891-1901. doi:10.1097/qad.0000000000000345

Keane, N. M., Roberts, S. G., Almeida, C. A. M., Krishnan, T., Chopra, A., Demaine, E., et al. (2012). High-avidity, high-IFN $\gamma$-producing CD8 T-cell Responses Following Immune Selection during HIV-1 Infection. Immunol. Cel Biol 90 (2), 224-234. doi:10.1038/icb.2011.34

Lehloenya, R. J., Peter, J. G., Copascu, A., Trubiano, J. A., and Phillips, E. J. (2020). Delabeling Delayed Drug Hypersensitivity: How Far Can You Safely Go? J. Allergy Clin. Immunol. Pract. 8 (9), 2878-2895. doi:10.1016/j.jaip.2020.07.005

Peter, J. G., Lehloenya, R., Dlamini, S., Risma, K., White, K. D., Konvinse, K. C., et al. (2017). Severe Delayed Cutaneous and Systemic Reactions to Drugs: A Global Perspective on the Science and Art of Current Practice. J. Allergy Clin. Immunol. Pract. 5 (3), 547-563. doi:10.1016/j.jaip.2017.01.025

Rozieres, A., Hennino, A., Rodet, K., Gutowski, M.-C., Gunera-Saad, N., Berard, F., et al. (2009). Detection and Quantification of Drug-specific T Cells in Penicillin Allergy. Allergy 64 (4), 534-542. doi:10.1111/j.13989995.2008.01674.x

Saade, F., Gorski, S. A., and Petrovsky, N. (2012). Pushing the Frontiers of T-Cell Vaccines: Accurate Measurement of Human T-Cell Responses. Expert Rev. Vaccin. 11 (12), 1459-1470. doi:10.1586/erv.12.125
TW010559-01. JT was supported by the Austin Medical Research Foundation (AMRF) and by a National Health and Medical Research Council (NHMRC) postgraduate scholarship (GNT 1139902) and Royal Australian College of Physicians Research Establishment Fellowship.

\section{SUPPLEMENTARY MATERIAL}

The Supplementary Material for this article can be found online at: https://www.frontiersin.org/articles/10.3389/fphar.2021.640012/ full\#supplementary-material

Sedgwick, J. D., and Holt, P. G. (1983). A Solid-phase Immunoenzymatic Technique for the Enumeration of Specific Antibody-Secreting Cells J. Immunol. Methods 57 (1-3), 301-309. doi:10.1016/0022-1759(83)90091-1

Sedlackova, L., Průcha, M., Poláková, I., and Míková, B., (2018). Evaluation of IFNGamma Enzyme-Linked Immunospot Assay (ELISPOT) as a First-Line Test in the Diagnosis of Non-immediate Hypersensitivity to Amoxicillin and Penicillin. Prague Med. Rep. 119 (1), 30-42. doi:10.14712/23362936.2018.3

Specian, A. F. L., Serpeloni, J. M., Tuttis, K., Ribeiro, D. L., Cilião, H. L., Varanda, E. A., et al. (2016). LDH, Proliferation Curves and Cell Cycle Analysis Are the Most Suitable Assays to Identify and Characterize New Phytotherapeutic Compounds. Cytotechnology 68 (6), 2729-2744. doi:10.1007/s10616-016-9998-6

Tanvarasethee, B., Buranapraditkun, S., and Klaewsongkram, J. (2013). The Potential of Using Enzyme-Linked Immunospot to Diagnose Cephalosporin-Induced Maculopapular Exanthems. Acta Derm Venerol 93 (1), 66-69. doi:10.2340/00015555-1386

Trubiano, J. A., Strautins, K., Redwood, A. J., Pavlos, R., Konvinse, K. C., Aung, A. K., et al. (2018). The Combined Utility of Ex Vivo IFN- $\gamma$ Release EnzymeLinked ImmunoSpot Assay and In Vivo Skin Testing in Patients with Antibiotic-Associated Severe Cutaneous Adverse Reactions. J. Allergy Clin. Immunol. Pract. 6 (4), 1287-1296. doi:10.1016/j.jaip.2017.09.004

Usui, T., Meng, X., Saide, K., Farrell, J., Thomson, P., Whitaker, P., et al. (2017). From the Cover: Characterization of Isoniazid-specific T-Cell Clones in Patients with Anti-tuberculosis Drug-Related Liver and Skin Injury. Toxicol. Sci. 155 (2), 420-431. doi:10.1093/toxsci/kfw218

Zembruski, N. C. L., Stache, V., Haefeli, W. E., and Weiss, J. (2012). 7Aminoactinomycin D for Apoptosis Staining in Flow Cytometry. Anal. Biochem. 429 (1), 79-81. doi:10.1016/j.ab.2012.07.005

Conflict of Interest: The authors declare that the research was conducted in the absence of any commercial or financial relationships that could be construed as a potential conflict of interest.

Publisher's Note: All claims expressed in this article are solely those of the authors and do not necessarily represent those of their affiliated organizations, or those of the publisher, the editors and the reviewers. Any product that may be evaluated in this article, or claim that may be made by its manufacturer, is not guaranteed or endorsed by the publisher.

Copyright (C) 2021 Copaescu, Choshi, Pedretti, Mouhtouris, Peter and Trubiano. This is an open-access article distributed under the terms of the Creative Commons Attribution License (CC BY). The use, distribution or reproduction in other forums is permitted, provided the original author(s) and the copyright owner(s) are credited and that the original publication in this journal is cited, in accordance with accepted academic practice. No use, distribution or reproduction is permitted which does not comply with these terms. 